\title{
Nonequilibrium spin distribution in single-electron transistor
}

\author{
Alexander N. Korotkov* and V. I. Safarov \\ GPEC, Departement de Physique, Faculté des Sciences de Luminy, Université de la \\ Méditerranée, 13288 Marseille, France
}

(February 23, 2017)

\begin{abstract}
Single-electron transistor with ferromagnetic outer electrodes and nonmagnetic island is studied theoretically. Nonequilibrium electron spin distribution in the island is caused by tunneling current. The dependencies of the magnetoresistance ratio $\delta$ on the bias and gate voltages show the dips which are directly related to the induced separation of Fermi levels for electrons with different spins. Inside a dip $\delta$ can become negative.
\end{abstract}

73.23.Hk; 75.70.Pa; 73.40.Rw

Typeset using REVTEX

*On leave from Nuclear Physics Institute, Moscow State University, Moscow 119899, Russia 
Magnetoresistance of tunnel structures is currently an attractive topic for both experimental and theoretical studies (see, e.g., Refs. [1-5]). For tunnel junctions made of ferromagnetic films, the difference as high as $26 \%$ at $4.2 \mathrm{~K}$ (up to $18 \%$ at room temperature) between the tunnel resistances for parallel and antiparallel film magnetization has been observed [2], that allows their application as magnetic sensors. The low temperature values agree well with the theoretical result [四] $\Delta R / R=2 P_{1} P_{2} /\left(1+P_{1} P_{2}\right)$ where $P_{1}$ and $P_{2}$ are spin polarizations of tunneling electrons in two films, that proves the good achieved quality of junctions. As an example, the polarization is about $47 \%$ for $\mathrm{CoFe}, 40 \%$ for $\mathrm{Fe}$, and $34 \%$ for $\mathrm{Co}$ [6, 2].

With the decrease of the tunnel junction area, the single-electron charging [7] becomes important leading to new physical effects. The study of tunnel magnetoresistance in this regime is a rapidly growing field [8 14] (see also Refs. [3] and [5]). For example, the enhancement of the magnetoresistance ratio $\Delta R / R$ due to Coulomb blockade has been discussed in Refs. [8, 13]. The magnetic field dependence of the tunneling current between two Co electrodes via the layer of few-nm Co clusters has been measured in Ref. [10]. In Ref. [12] the model of small ferromagnetic double tunnel junction (i.e. single-electron transistor [15] (SET-transistor) without gate electrode) has been considered, in which the tunnel resistances of junctions are different for parallel and antiparallel magnetizations of electrodes, thus changing the current through the system. A similar SET-transistor has been also studied theoretically in Ref. [14. The very interesting effect of magneto-Coulomb oscillations in SET-transistor has been observed and explained in Refs. [8,9]. Strong magnetic field $H$ causes the Zeeman shift of two spin bands and their repopulation. Since in ferromagnets the densities of states of these bands are different, the Fermi level moves with $H$ leading to magneto-Coulomb oscillations.

In the present letter we consider a SET-transistor which has ferromagnetic outer electrodes and nonmagnetic central island (see inset in Fig. 19a). When the coercive fields of two ferromagnetic electrodes are different, the standard technique of the magnetic field sweeping (see, e.g., Ref. [2]) easily allows to obtain parallel or antiparallel polarizations of outer 
electrodes. In the first approximation the current through SET-transistor does not depend on these polarizations because the island is nonmagnetic (the Zeeman splitting is negligible because of small $H$ ). However, if the electron spin relaxation in the island is not too fast (estimates are discussed later), then the tunneling of electrons with preferable spin orientation creates the nonequilibrium spin-polarized state of the island (similar to the effect discussed in Refs. [16,17] in absence of the Coulomb blockade). This in turn affects the tunneling in each junction and leads to different currents $I_{p}$ and $I_{a}$ through SET-transistor in the parallel and antiparallel configurations.

We will calculate the dependence of the relative current change $\delta=\left(I_{p}-I_{a}\right) / I_{p}$ on the bias and gate voltages (we call $\delta$ magnetoresistance ratio despite for finite voltage this terminology could be misleading). Nonzero $\delta$ is already the evidence of the nonequilibrium spin state in the island. Moreover, the voltage dependence of $\delta$ shows the dips, the width of which directly corresponds to the energy separation between Fermi levels of electrons with different spins in the island.

We consider SET-transistor consisting of two tunnel junctions with capacitances $C_{1}$ and $C_{2}$. Induced background charge $Q_{0}$ as usual [15] describes the influence of the gate voltage (general equivalence relations for finite gate capacitance are discussed, e.g., in Ref. [18]). We assume that the voltage scale related to the magnetic polarization of ferromagnetic electrodes [4] and the voltage scale of the barrier suppression [19] are large in comparison with the single-electron charging energy (that is a typical experimental situation). Then the polarization of outer electrodes can be taken into account by the difference between the tunnel resistances $R_{1,2}^{u}$ and $R_{1,2}^{d}$ for electrons with "up" and "down" spins. The total junction resistances $R_{1}=\left(1 / R_{1}^{u}+1 / R_{1}^{d}\right)^{-1}$ and $R_{2}=\left(1 / R_{2}^{u}+1 / R_{2}^{d}\right)^{-1}$ do not depend on the magnetic polarizations $P_{1}$ and $P_{2}$ of electrodes, while "partial" resistances are given by $R_{i}^{u}=2 R_{i} /\left(1+P_{i}\right)$ and $R_{i}^{d}=2 R_{i} /\left(1-P_{i}\right)$ (similar to the model of Ref. [四). Notice that $P_{i}$ describes the polarization of tunneling electrons [6] which is different (typically even in sign) from the total electron polarization at Fermi level (the latter one determines, e.g., the period of magneto-Coulomb oscillations [9]). 
We assume that the energy relaxation of electrons in the island is much faster than the spin relaxation. So, we characterize the nonequilibrium spin state by the difference $\Delta E_{F}$ between Fermi levels for "up" and "down" spins while both distributions are determined by the thermostat temperature $T$. The spin diffusion length is assumed to be much larger than the island size (that is a typical experimental situation - see Ref. [16]), so the spin distribution is uniform along the island.

The equations of the "orthodox" theory for single-electron transistor [7].15] (we assume $\left.R_{i} \gg R_{K}=h / e^{2}\right)$ should be modified in our case. The energy gain $W_{i}^{u(d) \pm}$ for tunneling to $(+)$ or from $(-)$ the island through $i$ th junction is different for "up" and "down" electrons,

$$
\begin{gathered}
W_{i}^{u \pm}(n)=W_{i}^{ \pm} \mp \frac{\Delta E_{F}}{2}, W_{i}^{d \pm}(n)=W_{i}^{ \pm} \pm \frac{\Delta E_{F}}{2} \\
W_{i}^{ \pm}=\frac{e}{C_{\Sigma}}\left[\mp\left(n e+Q_{0}\right) \mp(-1)^{i} \frac{V C_{1} C_{2}}{C_{i}}-\frac{e}{2}\right] .
\end{gathered}
$$

Here $n$ is the number of extra electrons on the island (as usual the electron is assumed to have positive charge $e$ ), $C_{\Sigma}=C_{1}+C_{2}$, and $V$ is the bias voltage. The corresponding tunneling rates satisfy usual equation [7]

$$
\Gamma_{i}^{s \pm}(n)=\frac{W_{i}^{s \pm}(n)}{e^{2} R_{i}^{s}\left(1-\exp \left(-W_{i}^{s^{ \pm}}(n) / T\right)\right)},
$$

where $s=u, d$ denotes spin. The average current $I$ through SET-transistor can be calculated as

$$
I=\sum_{n, s} e\left[\Gamma_{1}^{s+}(n)-\Gamma_{1}^{s-}(n)\right] \sigma(n)
$$

where $\sigma(n)$ is the stationary solution of the master equation [7]

$$
d \sigma(n) / d t=\sum_{i, s, \pm}\left[\sigma(n \pm 1) \Gamma_{i}^{s \mp}(n \pm 1)-\sigma(n) \Gamma_{i}^{s \pm}(n)\right] .
$$

Finally, the Fermi level separation $\Delta E_{F}$ should satisfy selfconsistent equation

$$
\begin{array}{r}
\Delta E_{F} \rho v / \tau \\
=\sum_{n, i}\left[\Gamma_{i}^{u+}(n)-\Gamma_{i}^{d+}(n)\right. \\
\left.-\Gamma_{i}^{u-}(n)+\Gamma_{i}^{d+}(n)\right] \sigma(n),
\end{array}
$$


where $\tau$ is the electron spin relaxation time for the island, $\rho$ is the density of states (per spin), and $v$ is island's volume. We introduce also the dimensionless spin relaxation time $\alpha=\tau / e^{2} \rho v\left(R_{1}+R_{2}\right)$.

The signs of polarizations $P_{1}$ and $P_{2}$ can be changed using the external magnetic field, that interchanges resistances $R_{i}^{u}$ and $R_{i}^{d}$. So the current $I_{p}$ for the parallel magnetization $\left(P_{1} P_{2}>0\right)$ is different from the current $I_{a}$ when one magnetization direction is reversed, $P_{2} \rightarrow-P_{2}$ (the change $P_{1} \rightarrow-P_{1}$ obviously gives the same result). Figure 11a shows the numerically calculated dependence of the magnetoresistance ratio $\delta$ (solid line) on the bias voltage $V$ for the SET-transistor with parameters $C_{1}=C_{2}, Q_{0}=0, T=0,\left|P_{1}\right|=\left|P_{2}\right|=$ $30 \%$, and $\alpha=0.1$. For the upper curve (shifted up for clarity) we assumed $R_{2}=R_{1}$ while $R_{2}=5 R_{1}$ for the lower curve. The $\delta-V$ dependence shows the oscillations with the same period $e / C_{i}$ as for the Coulomb staircase. The existence of oscillations is a trivial consequence of the charge dynamics in SET-transistor, similar to the effect discussed in Refs. [12,14].

More interesting features seen in Fig. 19a are the triangular-shape dips near the bias voltages

$$
V=\left[e / 2+n e+(-1)^{i} Q_{0}\right] / C_{i}
$$

at which the derivative of the I-V curve abruptly increases (the Coulomb staircase for $I_{p}$ shown in Fig. 1 1a by dashed lines is better seen for the lower curve). The edges of a dip correspond to the alignment between the Fermi level in an electrode and one of the split Fermi levels for electrons with different spins in the island. Hence, the dip width $\Delta V$ is directly related to the Fermi level splitting, $\Delta V=\Delta E_{F} C_{\Sigma} / e C_{i}$. Somewhat similar effect (the finite voltage caused by injected spin current) without Coulomb blockade had been observed in the "spin transistor" [16] fabricated using the thin film geometry with a size scale of $50 \mu \mathrm{m}$; the typical signal scale was only about $30 \mathrm{pV}$. The small size of the SETtransistor island leads to the very strong enhancement of the Fermi level separation (see Eq. (5)) and the corresponding voltage scale. 
The width of the dips in Fig. 1 1 a increases with voltage because the larger current provides larger $\Delta E_{F}$ (the crude estimate is $\left.\Delta E_{F}=\alpha I\left(P_{1}-P_{2}\right) e R_{\Sigma}\right)$. Actually in the case $\left|P_{1}\right|=\left|P_{2}\right|$ shown in the figures, the nonequilibrium spin distribution in the island occurs only for the antiparallel magnetization of electrodes; for parallel magnetization $\Delta E_{F}=0$ because the spin currents to/from different electrodes exactly cancel each other. When $\left|P_{1}\right| \neq\left|P_{2}\right|$ the complete cancellation does not occur, and the dip shape is determined by two different values of $\Delta E_{F}$ leading to the trapezoid-like shape instead of the triangular one.

It is interesting that the magnetoresistance ratio $\delta$ can be even negative within the dip range (see Fig. 1). This can be understood in the following way. The $I_{a}-V$ curve for the antiparallel magnetization generally goes below $I_{p}-V$ curve because the Fermi level splitting (which is larger in the antiparallel case) decreases the effective voltage for the spin band which provides the easier tunneling. However, this also splits the kinks on the I-V curve leading to the increase of $I_{a}$ (and decrease of $\delta$ ) within the splitting range. For sufficiently steep kink, $I_{a}$ can become even larger than $I_{p}$ (negative $\delta$ ). This also explains why the dips are more significant for larger tunnel resistance ratio (see Fig. 四a) when the Coulomb staircase is more pronounced.

Increase of the spin relaxation time $\tau$ leads to larger $\Delta E_{F}$ and, hence, increases $\delta$ as well as widens the dips, that is illustrated in Fig. 1 $1 \mathrm{~b}(\delta=0$ for $\tau=0)$. The change of the polarization amplitudes $\left|P_{1}\right|$ and $\left|P_{2}\right|$ leads to similar effects. Crudely, the magnetoresistance is determined by the product $\alpha\left|P_{1} P_{2}\right|$, while the exact shape of the $\delta-V$ curve depends on each of these parameters.

In the limit of large bias voltage the magnetoresistance ratio can be found analytically using the following expression for the current:

$$
\begin{aligned}
& I R_{\Sigma} / V=1-(\alpha / 2)\left(P_{1}-P_{2}\right)^{2} / \\
& {\left[1+(\alpha / 2)\left[R_{\Sigma}^{2} / R_{1} R_{2}-R_{1} R_{2}\left(P_{1} / R_{1}+P_{2} / R_{2}\right)^{2}\right]\right] .}
\end{aligned}
$$

However, the formula for $\delta$ is rather lengthy, so we present here only the result for small $\alpha$,

$$
\delta=2 \alpha\left|P_{1} P_{2}\right|,
$$


and the expression $\delta=2 \alpha\left|P_{1} P_{2}\right| /\left[1+2 \alpha\left(1-\left(\left|P_{1}\right|-\left|P_{2}\right|\right)^{2} / 4\right)\right]$ for the case $R_{1}=R_{2}$.

The finite temperature smears the features of the $\delta-V$ dependence (see Fig. 2a), but obviously does not change $\delta$ in the large-bias limit. The dips disappear when $T$ becomes comparable to $\Delta E_{F}$ while the oscillations disappear at higher temperatures determined by the single-electron energy scale $e^{2} / C_{\Sigma}$.

Notice that two series of dips determined by Eq. (6) coincide in Figs. 1 and 2a. With the change of the background charge $Q_{0}$ by the gate voltage, these two series will move in opposite directions. The dips can be also seen on the $\delta-Q_{0}$ dependence which is shown in Fig. 2 $2 \mathrm{~b}$ for different bias voltages $V$. The dip position moves with $V$. There are two dips per period, however, one of them is much less pronounced because of relatively large ratio of tunnel resistances.

To estimate the parameters of a possible experimental realization, let us assume $\mathrm{Co}-\mathrm{Cu}-$ Co SET-transistor. (Notice that Al-Co-Al SET-transistor has been already fabricated [9], however, aluminum is not suitable for our purpose because of its superconductivity.) The polarization $|P|=30 \%$ used in figures is a conservative value for Co. The spin relaxation rate $\tau$ for nonmagnetic island, which is the most crucial parameter of the effect, depends much on the material quality. In Ref. [20] $\tau \sim 10^{-7} \mathrm{~s}$ has been reported for very pure $\mathrm{Cu}$ at $T=1.4 \mathrm{~K}$ (the similar value has been found in Ref. [20 for Al, while $\tau \sim 10^{-8} \mathrm{~s}$ have been reported for $\mathrm{Al}$ in Ref. [16]). Let us choose $\tau=10^{-8} \mathrm{~s}$. Then using $\rho=9 \times 10^{21}$ $\mathrm{eV}^{-1} \mathrm{~cm}^{-3}$ for $\mathrm{Cu}, R_{\Sigma}=10^{5} \Omega$, and the island volume $v=200 \mathrm{~nm} \times 50 \mathrm{~nm} \times 20 \mathrm{~nm}$, we get $\alpha=0.35$. Hence, the effect of nonequilibrium spin distribution should be rather strong, and we could expect the magnetoresistance ratio $\delta$ up to $\sim 10 \%$ ( $\delta$ is significantly enhanced near the Coulomb blockade threshold - see Fig. 1). This large value allows to consider the possible applications of such a device. For $C_{\Sigma} \sim 3 \times 10^{-16} \mathrm{~F}$ the dips of the $\delta-V$ dependence could be observed at temperatures below $\sim 0.2 \mathrm{~K}$ while the oscillations could be noticeable up to $T \sim 1 \mathrm{~K}$.

In our theory we have neglected the Zeeman splitting $\pm g \mu_{B} H / 2$ because the typical coercive fields are relatively small, $H \sim 10^{2}$ Oe [2]. Hence the corresponding energy scale is 
very small, $\Delta E \sim 10^{-6} \mathrm{eV} \sim 10^{-2} \mathrm{~K}$, and the effect can hardly be observed.

We have discussed dc case only. If ac voltages are applied to the bias and/or gate electrodes, the similar formalism can be used. However, in this case the dynamic solution of the master equation (4) should be used instead of the stationary solution, and also the left side of Eq. (5) should be replaced by $\left[d\left(\Delta E_{F}\right) / d t+\Delta E_{F} / \tau\right] \rho v$. The nontrivial dependence starts when the frequency $f$ of the applied voltage becomes comparable to $\tau^{-1}$. These frequencies are within the experimentally achievable range, so such an experiment could be used for the direct measurement of the spin relaxation time $\tau$.

In conclusion, we have considered the SET-transistor consisting of ferromagnetic electrodes and nonmagnetic island. We have predicted that the nonequilibrium spin distribution in the island leads to a considerable magnetoresistance which has a specific dependence on the bias and gate voltages. In particular, it shows the dips directly related to the Fermi level splitting.

The authors thank D. V. Averin and K. K. Likharev for useful discussions. A. K. has been supported in part by French MENRT (PAST), Russian RFBR, and Russian Program on Nanoelectronics. 


\section{REFERENCES}

[1] M. Julliere, Phys. Lett. A 54, 225 (1975).

[2] J. S. Moodera, L. R. Kinder, T. M. Wong, and R. Merservey, Phys. Rev. Lett. 74, 3273 (1995); J. S. Moodera and L. R. Kinder, J. Appl. Phys. 79, 4724 (1996).

[3] J. S. Helman and B. Abeles, Phys. Rev. Lett. 37, 1429 (1976).

[4] J. C. Slonczewski, Phys. Rev. B 39, 6995 (1989).

[5] A. Milner, A. Gerber, B. Groisman, M. Karpovsky, and A. Gladkikh, Phys. Rev. Lett. 76, 475 (1996).

[6] R. Meservey and P. M. Tedrow, Phys. Rep. 238, 173 (1994).

[7] D. V. Averin and K. K. Likharev, in Mesoscopic Phenomena in Solids, edited by B. L. Altshuler, P. A. Lee, and R. A. Webb (Elsevier, Amsterdam, 1991), p. 173.

[8] K. Ono, H. Shimada, and Y. Ootuka, J. Phys. Soc. Jpn. 66, 1261 (1997).

[9] K. Ono, H. Shimada, and Y. Ootuka, cond-mat/9803070.

[10] L. F. Schelp, A. Fert, F. Fettar, P. Holody, S. F. Lee, J. L. Maurice, F. Petroff, and A. Vaurès, Phys. Rev. B 56, R5747 (1997).

[11] S. Sankar, B. Dieny, and A. E. Berkowitz, J. Appl. Phys. 81, 5512 (1997).

[12] J. Barnaś and A. Fert, Phys. Rev. Lett. 80, 1058 (1998).

[13] S. Takahashi and S. Maekawa, Phys. Rev. Lett. 80, 1758 (1998).

[14] K. Majumdar and S. Hershfield, cond-mat/9709206.

[15] K. K. Likharev, IEEE Trans. on Magn. 23, 1142 (1987).

[16] M. Johnson and R. H. Silsbee, Phys. Rev. B 37, 5326 (1988).

[17] M. Johnson, Phys. Rev. Lett. 70, 2142 (1993). 
[18] A. N. Korotkov, R. H. Chen, and K. K. Likharev, J. Appl. Phys. 78, 2520 (1995).

[19] A. N. Korotkov and Yu. V. Nazarov, Physica B 173, 217 (1991).

[20] D. Lubzens and S. Schultz, Phys. Rev. Lett. 36, 1104 (1976). 


\section{FIGURES}

FIG. 1. The dependence of the magnetoresistance ratio $\delta$ on the bias voltage $V$ for (a) two different ratios $R_{2} / R_{1}$ and (b) for several values of the dimensionless spin relaxation time $\alpha$. Inset in (a) shows the schematic of the SET-transistor with ferromagnetic (F) outer electrodes and nonmagnetic $(\mathrm{N})$ island, while the dashed lines show the $I_{p}-V$ curves (arbitrary units).

FIG. 2. (a) The $\delta-V$ dependence for different temperatures $T$ and (b) the dependence of $\delta$ on the background charge $Q_{0}$ for several bias voltages. 

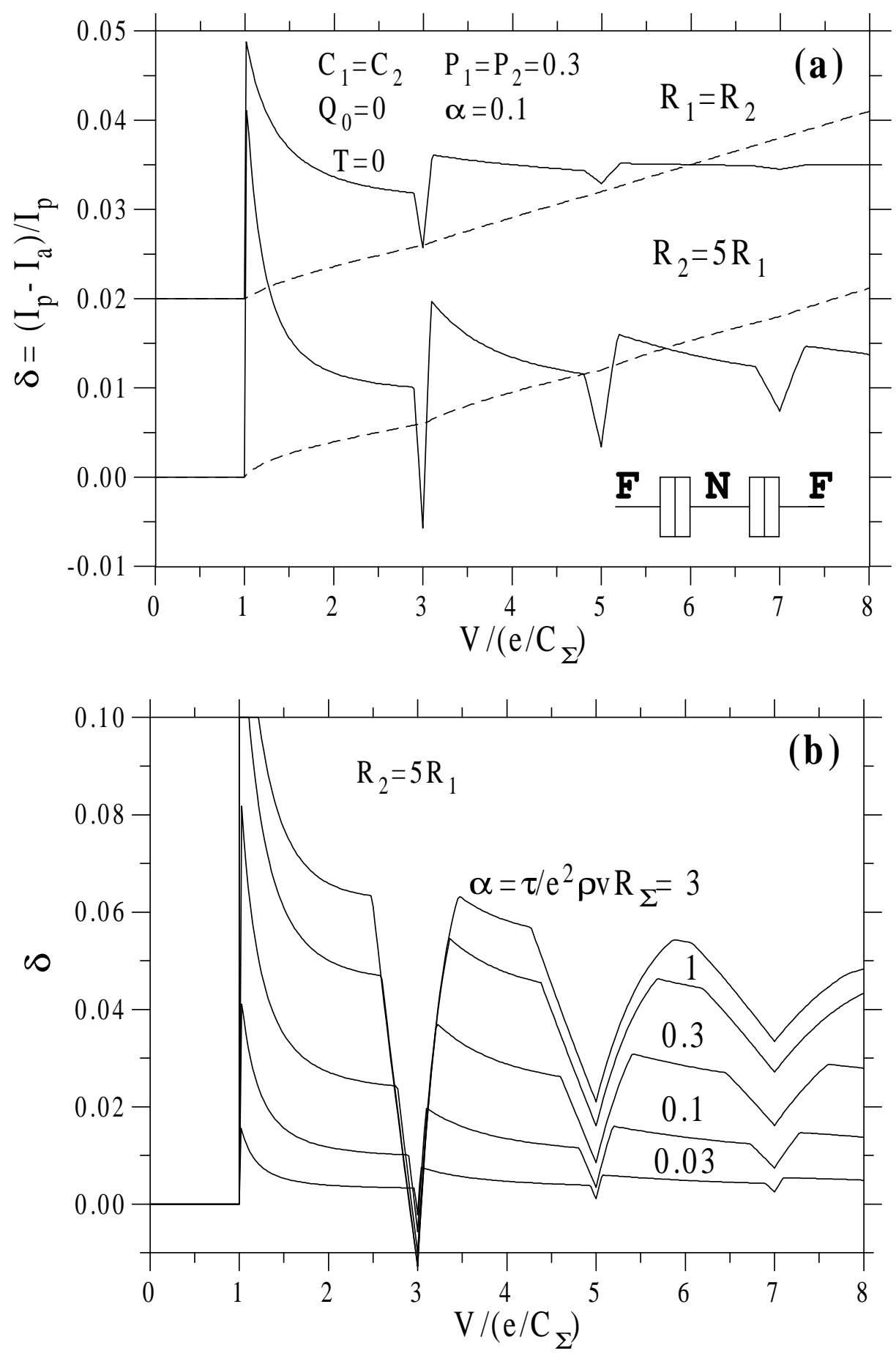

Fig. 1 

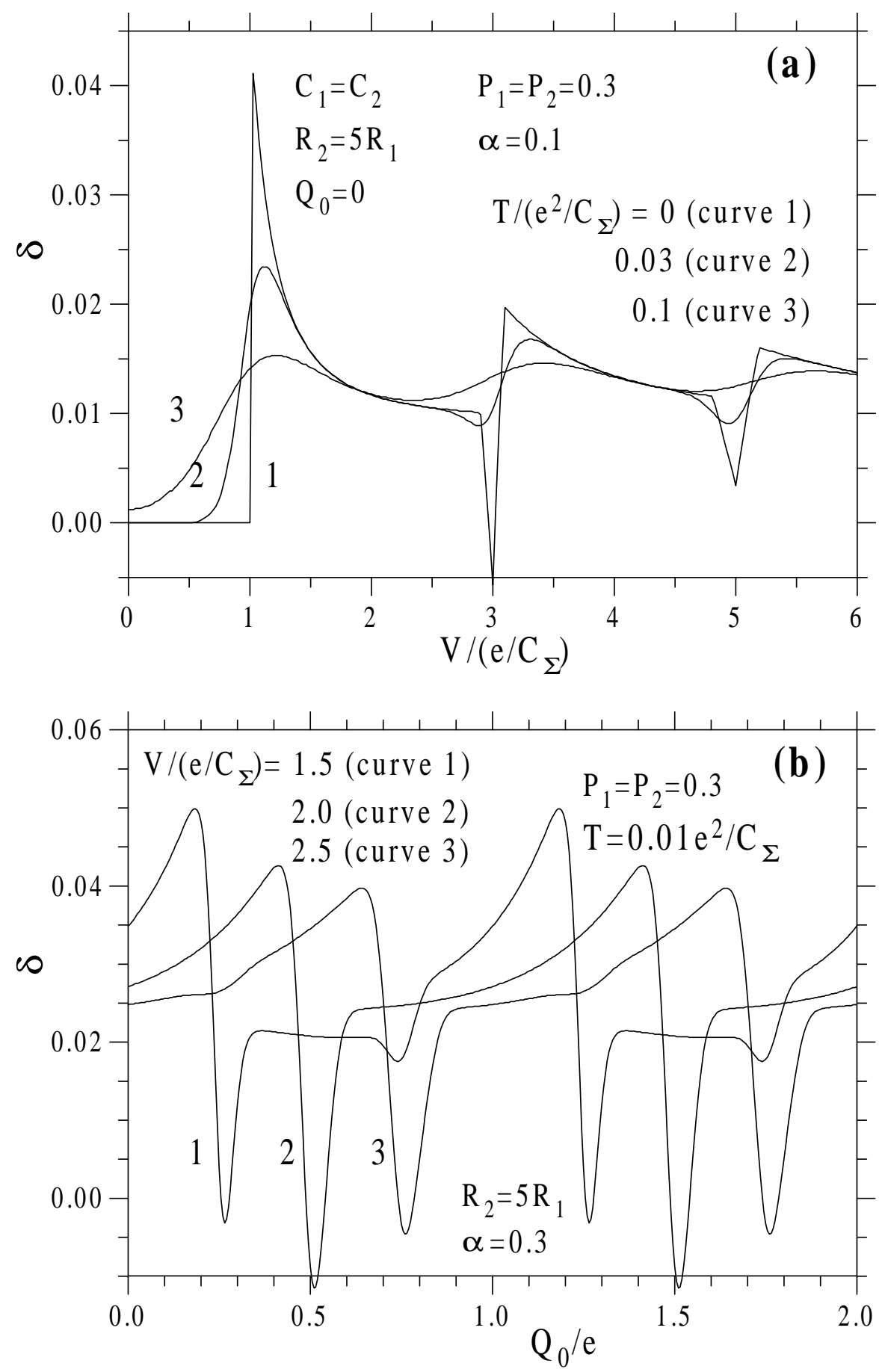